\title{
Long-term results of aggressive hemiarch replacement in 534 patients with type A aortic dissection
}

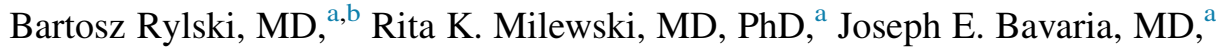 \\ Prashanth Vallabhajosyula, MD, ${ }^{\mathrm{a}}$ William Moser, CRNP, ${ }^{\mathrm{a}}$ Wilson Y. Szeto, MD, ${ }^{\mathrm{a}}$ and \\ Nimesh D. Desai, MD, PhD
}

Objective: To present the outcomes of routinely performed hemiarch replacement in patients with acute type A aortic dissection.

\begin{abstract}
Methods: From 1993 to 2013, among 629 patients with acute type A dissection, 534 patients (85\%) underwent hemiarch, 63 patients $(10 \%)$ underwent hemiarch and antegrade thoracic stent grafting, 26 patients $(4 \%)$ underwent total arch, and 6 patients $(1 \%)$ underwent isolated ascending replacement. Patients with hemiarch replacement comprised the study population. Median follow-up was 4.1 years (first quartile, 1.9; third quartile, 7.8) (2462 patient years).

Results: In-hospital mortality was $12 \%$ (66 out of 534 patients). Survival was $80 \% \pm 2 \%, 68 \% \pm 3 \%$, and $51 \% \pm 3 \%$, and $84 \% \pm 3 \%, 65 \% \pm 4 \%$, and $41 \% \pm 6 \%$ in DeBakey type I and II patients at 1,5 , and 10 years, respectively ( $\log$ rank $P=.375$ ). Freedom from distal aortic reintervention was $97 \% \pm 1 \%, 90 \% \pm$ $2 \%$, and $85 \% \pm 3 \%$ and $99 \% \pm 1 \%, 97 \% \pm 2 \%$, and $90 \% \pm 5 \%$ in DeBakey type I and II patients at 1 , 5 , and 10 years, respectively ( $\log$ rank $P=.046)$. Seven patients $(1.3 \%)$ required reintervention for aortic arch aneurysm and 25 patients $(5 \%)$ required reintervention for descending aortopathy. The success rate for distal reintervention performed electively or urgently was $92 \%$ (24 out of 26 patients). Marfan syndrome (odds ratio, 3.43; $P=.046$ ) and DeBakey type I dissection (odds ratio, $2.49 ; P=.048$ ) were independent predictors of distal aortic reintervention.
\end{abstract}

Conclusions: Aggressive hemiarch replacement in acute type A dissection can be performed with low mortality and low aortic arch reoperation rate. Resection of all dissected aortic wall tissue decreases, but does not eliminate, the risk of later adverse aortic events. (J Thorac Cardiovasc Surg 2014;148:2981-5)

In patients with acute Stanford type A aortic dissection optimal surgery for the aortic arch is still a matter of debate. Standard treatment options, including open distal anastomosis, hemi- or total arch replacement, and frozen elephant trunk have recently been enriched by the hybrid approach; that is, combining surgical replacement of the aortic arch with antegrade endovascular repair of the descending aorta. $^{1,2}$ The current American College of Cardiology Foundation guidelines recommend, in patients with type A dissection, aortic arch replacement in case of arch aneurysm and replacement of the entire dissected aorta if a DeBakey type II dissection is present. ${ }^{3}$ Nonetheless, decision on surgical technique is usually based on institutional experience or surgeon preferences.

\footnotetext{
From the Division of Cardiovascular Surgery, ${ }^{\text {a }}$ Hospital of the University of Pennsylvania, Philadelphia, Pa; and Heart Center Freiburg University, ${ }^{\mathrm{b}}$ Freiburg, Germany.

Disclosures: Authors have nothing to disclose with regard to commercial support. Read at the American Association for Thoracic Surgery Aortic Symposium 2014, New York, New York, April 24-25, 2014.

Received for publication April 3, 2014; revisions received May 18, 2014; accepted for publication May 21, 2014; available ahead of print Aug 10, 2014.

Address for reprints: Bartosz Rylski, MD, Heart Center Freiburg University, Hugstetter St 55, 79106 Freiburg, Germany (E-mail: bartosz.rylski@universitaets-herzzentrum.de). $0022-5223 / \$ 36.00$

Copyright (c) 2014 by The American Association for Thoracic Surgery http://dx.doi.org/10.1016/j.jtcvs.2014.05.093
}

The aim of our investigation was to delineate long-term outcomes of standardized aggressive hemiarch replacement in patients with acute type A aortic dissection at a single institution and to examine the hypothesis that complete resection of a dissected aorta, if feasible, is curative.

\section{PATIENTS AND METHODS}

\section{Study Population and Definitions}

Between 1993 and 2013, among 629 patients who underwent ascending aortic replacement for acute Stanford type A aortic dissection, 534 (85\%) underwent aggressive hemiarch, 63 patients $(10 \%)$ underwent aggressive hemiarch with antegrade thoracic stent grafting, 26 patients underwent $(4 \%)$ total arch, and 6 patients $(1 \%)$ underwent isolated ascending aortic replacement. Patients who underwent aggressive hemiarch replacement (without antegrade thoracic stent grafting) comprise the study population

For our study, acute aortic dissection was defined as a dissection operated on no later than 14 days after the symptom onset. Malperfusion syndromes were diagnosed by clinical examination and confirmed by computed tomography angiography and were defined as altered blood flow with clinical evidence of a lack of blood flow resulting in ischemia with organ dysfunction. Cardiogenic shock was defined as persistent hypotension (systolic blood pressure $<80 \mathrm{~mm} \mathrm{Hg}$ ) with severe reduction in cardiac index. Our institutional review committee approved this retrospective study and the need for informed consent was waived.

\section{Demographics and Clinical Presentation}

Clinical presentation details are summarized in Table 1 . The average age was 62 years (first quartile, 51; third quartile, 73). Fifty-six patients 


\section{Abbreviation and Acronym \\ TAAA $=$ thoracoabdominal aortic aneurysm}

$(9 \%)$ were $>80$ years old. Most patients were men $(64 \%)$. Bicuspid aortic valve was observed in $35(7 \%)$ and Marfan syndrome in $25(5 \%)$ patients. Forty-seven patients $(9 \%)$ underwent previous cardiac surgery. Malperfusion syndrome was evident in 161 patients $(30 \%)$ and 122 patients $(23 \%)$ were operated on in cardiogenic shock. DeBakey type I dissection was observed in 357 patients $(67 \%)$.

\section{Surgical Approach}

In all patients previously described, a standardized integrated surgical management was applied. ${ }^{4}$ This approach included direct admission to the operating room, immediate operative repair, replacing the entire ascending aorta, resuspension of the aortic valve and Teflon (Dupont, Wilmington, Del) felt neomedia root repair (or root replacement in case of sinus segment aneurysm or intimal tear extending beyond the sinotubular junction). In patients with intimal tear along the greater aortic curvature total aortic arch replacement was performed; all others underwent a relatively aggressive, extended hemiarch replacement with excision of most of the diseased aortic arch and distal anastomosis sewn at opposite the origin of the left subclavian artery. Residual arch tissue was reinforced with Teflon felt neomedia. In most patients the Teflon felt repair was secured with a small amount of BioGlue (CryoLife Inc, Kennesaw, $\mathrm{Ga}$ ) between the dissected layers.

The aortic arch was replaced in an open fashion and in hypothermic arrest. Cerebral protection was achieved in $90 \%$ of patients by means of retrograde and in $10 \%$ by selective antegrade cerebral perfusion. In those with retrograde perfusion requiring more extensive arch reconstruction necessitating $>40$ minutes of circulatory arrest, selective bilateral antegrade cerebral perfusion was used by balloon-tipped cannulas placed in both the innominate and left common carotid arteries. When aortic arch reconstruction was completed, a Dacron graft was cannulated to resume antegrade cardiopulmonary bypass and start the rewarming process. Surgical details, cannulation sites for arterial inflow, and perfusion times are summarized in Table 2.

\section{Patient Follow-up}

Surveillance follow-up data were obtained by contacting the patients' general practitioners, the patients, and their family members; from aortic clinic office visits; or by the Social Security Death Index. Complete follow-up was available in 451 of 468 hospital survivors (98\%). Patients were followed up a total of 2462 patient years, with a median follow-up among survivors of 4.1 years (first quartile, 1.9; third quartile, 7.8 ), ranging from 0.5 to 20 years. Eighteen percent were followed for 10 or more years.

In accordance with current guidelines, ${ }^{3}$ the follow-up protocol included postoperative computed tomography angiography before discharge, clinical examination, and computed tomography angiography 6 and 12 months postoperatively and annually thereafter at our institutional aortic outpatient clinic (or at their regional hospitals for patients living in remote areas).

\section{Statistical Analysis}

Continuous data are presented as median (first quartile and third quartile); categorical variables are given as counts and percentages. Survival was analyzed using the Kaplan-Meier method and log rank calculations. Multivariable logistic regression was performed to analyze the influences of age $<45$ years, bicuspid aortic valve syndrome, Marfan syndrome, and DeBakey type I dissection on distal aortic reintervention rates.
TABLE 1. Demographics and clinical presentation $(N=534)$

\begin{tabular}{lc}
\hline \multicolumn{1}{c}{ Characteristic } & Result \\
\hline Age, y & $62(51,73)$ \\
Age $>80$ y & $56(9)$ \\
Male gender & $344(64)$ \\
Clinical presentation & \\
Current smoker & $13(2)$ \\
Hypertension & $423(79)$ \\
Diabetes mellitus & $46(9)$ \\
Previous cardiac surgery & $47(9)$ \\
$\quad$ Previous coronary artery bypass grafting & $32(6)$ \\
$\quad$ Previous aortic valve replacement & $10(2)$ \\
Chronic obstructive pulmonary disease & $52(10)$ \\
Renal failure & $44(8)$ \\
Coronary artery disease & $95(18)$ \\
Prior stroke & $41(8)$ \\
Prior transient ischemic attack & $26(5)$ \\
Marfan syndrome & $25(5)$ \\
Bicuspid aortic valve & $35(7)$ \\
Extent of dissection & \\
DeBakey type I & $357(67)$ \\
DeBakey type II & $177(33)$ \\
Ascending aortic diameter, cm & $5.0(4.4,5.7)$ \\
Moderate-severe aortic valve insufficiency & $181(34)$ \\
Cardiac tamponade & $133(25)$ \\
Cardiogenic shock & $122(23)$ \\
Malperfusion & \\
$\geq 1$ Organ & $161(30)$ \\
Coronary & $33(6)$ \\
Cerebral & $47(9)$ \\
Iliofemoral & $71(13)$ \\
Gastrointestinal & $27(5)$ \\
\hline Cpinal & $7(1)$ \\
\hline
\end{tabular}

Categorical values are given as $\mathrm{n}(\%)$, continuous values are median (first quartile, third quartile).

\section{RESULTS}

\section{In-Hospital Mortality and Survival}

Postoperative complications are listed in Table 2. The inhospital mortality was $12 \%$ (66 of 534 patients). The primary reason for in-hospital death was multisystem organ failure in 37 patients $(56 \%)$, heart failure in 17 patients $(26 \%)$, exsanguination in 4 patients $(6 \%)$, neurologic events in 5 patients $(8 \%)$, and descending aortic rupture in 3 patients $(4 \%)$. Overall survival was $82 \% \pm 2 \%$, $68 \% \pm 3 \%$, and $51 \% \pm 3 \%$ in patients with DeBakey type I and $84 \% \pm 3 \%, 65 \% \pm 4 \%$, and $41 \% \pm 6 \%$ in patients with DeBakey type II dissection at 1,5 , and 10 years with 55 and 20 patients remaining at risk at 10 years, respectively (log rank $P=.375$ ) (Figure 1 ).

\section{Distal Aortic Reinterventions}

Freedom from distal reintervention was $97 \% \pm 1 \%$, $90 \% \pm 2 \%$, and $85 \% \pm 3 \%$ in patients with DeBakey type $\mathrm{I}$ and $99 \% \pm 1 \%, 97 \% \pm 2 \%$, and $90 \% \pm 5 \%$ in 
TABLE 2. Details and outcome for surgery for type A aortic dissection $(\mathbf{N}=\mathbf{5 3 4})$

\begin{tabular}{lc}
\hline \multicolumn{1}{c}{ Surgical detail } & Outcome \\
\hline Proximal repair & \\
Aortic valve resuspension* & $414(78)$ \\
Aortic root replacement & $103(19)$ \\
Valve sparing aortic root replacement & $6(1)$ \\
Wheat procedure $\dagger$ & $12(2)$ \\
Arterial cannulation site & \\
Femoral artery & $301(56)$ \\
Ascending aorta & $179(34)$ \\
Axillary artery & $46(9)$ \\
Innominate artery & $8(1)$ \\
Cardiopulmonary bypass time, min & $205(182,247)$ \\
Crossclamp time, min & $135(115,166)$ \\
Hypothermic circulatory arrest time, min & $32(25,40)$ \\
Outcome & \\
Chest reexploration for bleeding & $36(7)$ \\
New stroke & $27(5)$ \\
Transient ischemic attack & $26(5)$ \\
Acute renal failure & $76(14)$ \\
Temporary dialysis & $41(8)$ \\
In-hospital mortality & $66(12)$ \\
\hline Categorical values are given as n (\%), continuous values are median (first quartile, \\
third quartile). *Aortic valve resuspension includes supracoronary ascending replace- \\
ment, sinus of Valsalva repair with a Teflon felt neomedia (Dupont, Wilmington, Del), \\
and valve resuspension in patients with native aortic valve. $\dagger$ Wheat procedures in- \\
cludes aortic valve replacement, sinus of Valsalva repair with a Teflon felt neomedia, \\
and supracoronary ascending replacement.
\end{tabular}

patients with DeBakey type II dissection at 1,5 , and 10 years, respectively ( $\log$ rank $P=.046$ ) (Figure 2). Thirtytwo $(6 \%)$ patients inclusive of 4 Marfan syndrome and 6 DeBakey type II patients at median 3.0 years (first quartile,

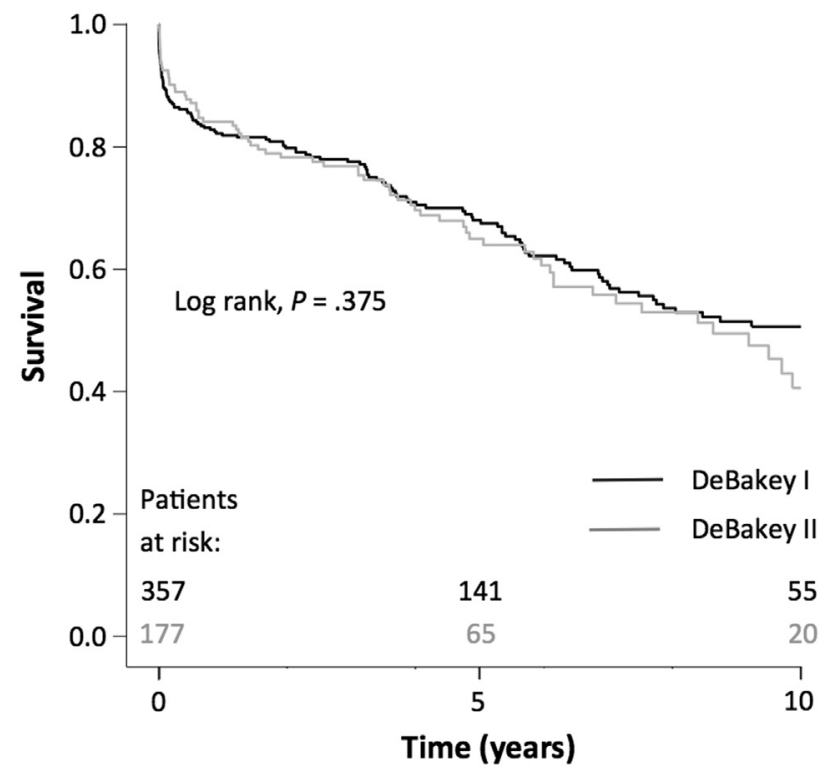

FIGURE 1. Kaplan-Meier analysis of overall survival in patients with acute DeBakey type I and II aortic dissection.

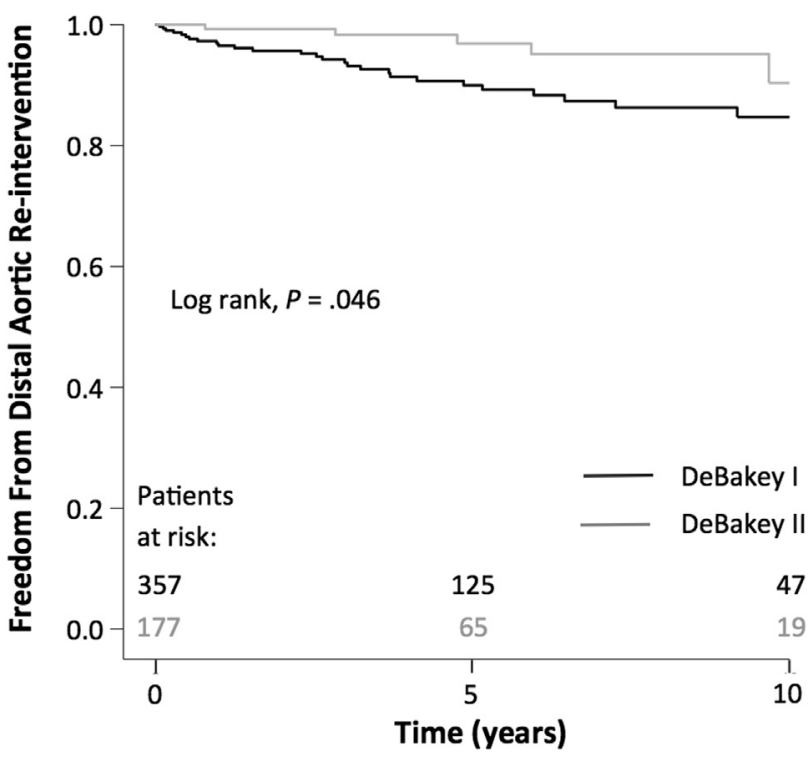

FIGURE 2. Kaplan-Meier analysis of freedom from aortic distal reintervention in patients with acute DeBakey type I and II aortic dissection.

1.0; third quartile, 5.2) postoperatively required distal reinterventions: 18 patients $(56 \%)$ for thoracoabdominal aortic aneurysm (TAAA) Crawford type I, 6 patients $(19 \%)$ for TAAA type II, 1 patient $(3 \%)$ for TAAA type III, 5 patients $(16 \%)$ for pseudoaneurysm at distal anastomosis, and 2 patients $(6 \%)$ for aortic arch aneurysm. Overall, 28 patients underwent open aortic repair and in 4 patients the endovascular approach was applied. Aortic reintervention was performed emergently (immediately after admission) in 6 patients ( 2 with ruptured new-onset type B dissection and 4 with ruptured dissected aneurysm) and urgently (ie, within 24 hours after admission) in 3 patients. Six patients did not survive the surgery or the early postoperative phase and among them 4 patients were operated on emergently. The overall success rate for distal reintervention performed electively or urgently was $92 \%$ (24 of 26 patients).

\section{Distal Aortic Reintervention Risk Factors}

Marfan syndrome (OR, 3.43; $P=.046)$ and DeBakey type I dissection (OR, 2.49; $P=.048)$ were independent predictors of distal aortic reintervention (Table 3).

TABLE 3. Multivariable analysis of distal aortic reintervention risk factors

\begin{tabular}{lccc}
\hline \multicolumn{1}{c}{ Variable } & $\begin{array}{c}\text { Odds } \\
\text { ratio }\end{array}$ & $\begin{array}{c}\mathbf{9 5 \%} \text { Confidence } \\
\text { interval }\end{array}$ & $\boldsymbol{P}$ value \\
\hline Age $<45$ y & 1.33 & $0.50-3.50$ & .566 \\
Bicuspid aortic valve & 0.39 & $0.05-3.00$ & .367 \\
Marfan syndrome & 3.43 & $1.02-11.5$ & .046 \\
DeBakey type I aortic dissection & 2.49 & $1.01-6.14$ & .048 \\
\hline
\end{tabular}


Age $<45$ years (OR, 1.33; $P=.566)$ was not associated with aortic reintervention at follow-up.

\section{DISCUSSION}

In the setting of acute type A aortic dissection, the primarily aim of the surgery is preserving life by preventing aortic rupture, correcting aortic valve insufficiency, and restoring flow to dissected branch vessels. There is a general consensus that addressing the aortic arch during the primary surgery is necessary, but only in case of arch aneurysm or intimal tear located in the arch. Whereas several groups reported on increased perioperative mortality and stroke rates in patients undergoing more aggressive arch replacement ${ }^{5,6}$ with the rate of reoperation not affected by the type of arch surgery, ${ }^{7}$ others did not identify extended replacement into the aortic arch as a perioperative risk factor. ${ }^{8,9}$ According to our algorithm introduced 20 years ago, ${ }^{4}$ we perform, in patients with acute type A dissection, aggressive hemiarch replacement with resection of the entire lesser curvature and most of the dissected aortic arch wall. Only in case of intimal tear along the greater curvature is total aortic arch replacement performed. Despite this more aggressive distal aortic surgical approach, in-hospital mortality was $12 \%$, corresponding to the lower end of the range in published series. ${ }^{10-12}$

Hemiarch replacement was associated with low reoperation rate for aortic arch aneurysm. Only 2 patients $(0.4 \%)$ revealed an arch aneurysm and $5(1 \%)$ developed pseudoaneurysm at the distal anastomosis in the cohort of 534 patients. Most of our descending aortic reinterventions were performed for TAAA Crawford type I. Elective aortic reinterventions were performed safely in $92 \%$ of patients; however, among 6 patients who presented with ruptured descending aorta, 4 did not survive the surgery. These data underline the importance of close follow-up of all patients with type A aortic dissection, particularly of those with Marfan syndrome and DeBakey type I dissection because both of these conditions were independent predictors of distal aortic reintervention. Most reinterventions were performed in an open fashion. Endovascular repair of chronic aortic dissection is controversial. In the early study period we reoperated on patients with chronic dissection exclusively in an open fashion. In later study years, use of endovascular techniques became more common and currently we consider the endovascular approach, especially in older patients with multimorbidities.

Aggressive hemiarch replacement, as a treatment of choice in patients with type A dissection, allows for resection of all dissected tissue in patients with DeBakey type II dissection. Based on the suggestion that complete resection of a dissected aorta could be curative, ${ }^{2}$ we analyzed the outcomes stratified to patients with and without remaining dissected aorta (DeBakey type I and II). We did not observe any significant differences in in-hospital mortality and in the overall survival between groups. Patients with complete resection of a dissected aorta (DeBakey type II) had significantly fewer aortic distal reinterventions, but were not free of aortic adverse events requiring surgery: 2 presented with ruptured new-onset type B dissection, 3 had TAAA type I, and 1 patient had pseudoaneurysm at the distal anastomosis. According to these results, complete resection of all dissected aorta may not be considered a curative aortic treatment. Aortic dissection seems to be an indicator of disease of the entire aorta. Elimination of a dissected aortic segment does not eliminate aortic disease. Long-term follow-up is essential in the entire type A dissection population regardless the dissection extension.

\section{CONCLUSIONS}

Aggressive hemiarch aortic replacement in the setting of acute Stanford type A aortic dissection can be performed with low in-hospital mortality, low perioperative stroke rates, and low aortic arch reoperation rate. Resection of all dissected aortic wall tissue decreases but does not eliminate the risk of later adverse aortic events. The overall long-term survival in type A aortic dissection is not influenced by the replacement of all dissected aortic segments by the initial surgery.

\section{References}

1. Pochettino A, Brinkman WT, Moeller P, Szeto WY, Moser W, Cornelius K, et al. Antegrade thoracic stent grafting during repair of acute DeBakey I dissection prevents development of thoracoabdominal aortic aneurysms. Ann Thorac Surg. 2009;88:482-9.

2. Tsagakis K, Tossios P, Kamler M, Benedik J, Natour D, Eggebrecht H, et al. The DeBakey classification exactly reflects late outcome and re-intervention probability in acute aortic dissection with a slightly modified type II definition. Eur J Cardiothorac Surg. 2011;40:1078-84.

3. Hiratzka LF, Bakris GL, Beckman JA, Bersin RM, Carr VF, Casey DE, et al. 2010 ACCF/AHA/AATS/ACR/ASA/SCA/SCAI/SIR/STS/SVM guidelines for the diagnosis and management of patients with Thoracic Aortic Disease: a report of the American College of Cardiology Foundation/American Heart Association Task Force on Practice Guidelines, American Association for Thoracic Surgery, American College of Radiology, American Stroke Association, Society of Cardiovascular Anesthesiologists, Society for Cardiovascular Angiography and Interventions, Society of Interventional Radiology, Society of Thoracic Surgeons, and Society for Vascular Medicine. Circulation. 2010;121:e266-369.

4. Bavaria JE, Pochettino A, Brinster DR, Gorman RC, McGarvey ML, Gorman JH, et al. New paradigms and improved results for the surgical treatment of acute type A dissection. Ann Surg. 2001;234:336-42.

5. Ohtsubo S, Itoh T, Takarabe K, Rikitake K, Furukawa K, Suda H, et al. Surgical results of hemiarch replacement for acute type A dissection. Ann Thorac Surg. 2002;74(Suppl):S1853-6.

6. Pagni S, Ganzel BL, Trivedi JR, Singh R, Mascio CE, Austin EH, et al. Early and midterm outcomes following surgery for acute type a aortic dissection. J Card Surg. 2013;28:543-9.

7. Kim JB, Chung CH, Moon DH, Ha GJ, Lee TY, Jung SH, et al. Total arch repair versus hemiarch repair in the management of acute DeBakey type I aortic dissection. Eur J Cardiothorac Surg. 2011;40:881-7.

8. Tan ME, Dossche KM, Morshuis WJ, Kelder JC, Waanders FG, Schepens MA. Is extended arch replacement for acute type a aortic dissection an additional risk factor for mortality? Ann Thorac Surg. 2003;76:1209-14.

9. Easo J, Weigang E, Hölzl PP, Horst M, Hoffmann I, Blettner M, et al. Influence of operative strategy for the aortic arch in DeBakey type I aortic dissection: analysis of the German Registry for Acute Aortic Dissection Type A. J Thorac Cardiovasc Surg. 2012;144:617-23. 
10. Rylski B, Beyersdorf F, Blanke P, Boos A, Hoffmann I, Dashkevich A, et al. Supracoronary ascending aortic replacement in patients with acute aortic dissection type A: What happens to the aortic root in the long run? J Thorac Cardiovasc Surg. 2013;146:285-90.

11. Rylski B, Hoffmann I, Beyersdorf F, Suedkamp M, Siepe M, Nitsch B, et al. Acute aortic dissection type a: age-related management and outcomes reported in the German Registry for Acute Aortic Dissection Type A (GERAADA) of over 2000 patients. Ann Surg. 2014;259:598-604.

12. Dell'aquila AM, Concistrè G, Gallo A, Pansini S, Piccardo A, Passerone G, et al. Fate of the preserved aortic root after treatment of acute type A aortic dissection: 23-year follow-up. J Thorac Cardiovasc Surg. 2013;146: 1456-60. 